\title{
How to balance the development of new surgical techniques and protection of patients' health interests
}

\author{
Xiao-Ping Geng \\ Hepatobiliary Surgery Department, The First Affiliated Hospital of Anhui Medical University, Hefei 230022, China \\ Correspondence to: Xiao-Ping Geng. The First Affiliated Hospital of Anhui Medical University, Hefei 230022, China. Email: xp_geng@163.net. \\ Provenance and Peer Review: This article was commissioned by the editorial office, Hepatobiliary Surgery and Nutrition. The article did not undergo \\ external peer review.
}

Submitted Mar 31, 2020. Accepted for publication Apr 23, 2020.

doi: $10.21037 /$ hbsn.2020.04.09

View this article at: http://dx.doi.org/10.21037/hbsn.2020.04.09

In 1992, when the usage of laparoscopic cholecystectomy began to rise, Altman (1) published "When Patient's Life Is Price of Learning New Kind of Surgery" in The New York Times. Altman pointed out that accidental injuries to patients during the rapid development of new surgical techniques were often overlooked and attributed to the price of the "learning curves" attached to these techniques. He emphasized that surgeons should only carry out laparoscopic cholecystectomy when they are confident in performing traditional open cholecystectomy. Thirty years have passed, and Altman's warning still seems valuable.

Since the first successful laparoscopic cholecystectomy in 1988, the laparoscopic devices have developed to being $4 \mathrm{~K}$ Ultra HD, 3D, and fluorescent display under the help from industry. New surgical systems like the Da Vinci have also attracted more attention from surgeons in using these new techniques, which have now been applied to many different kinds of complicated surgeries, like pancreatoduodenectomy, liver lobectomy, and hilar cholangiocarcinoma resection. The problems, however, remain. How can we avoid paying the price of harming a patient's health or life while developing new techniques?

First, we hypothesize that traditional surgical training is the foundation before any new techniques can be promoted. In traditional surgical training, surgeons often first perform animal experiments, then are aided by senior surgeons for more practice on the table before masting surgery independently. This pathway enables surgeons to gain a better understanding of the anatomy, the problematic parts of an operation, and the flow of various strategies in the open surgery field, which is the core of surgical training.
Only after this pathway, can surgeons confidently adopt new technologies, such as laparoscopy or robotic surgery, which involve little tactile sensation, while reducing the "learning curve" of harm to the patients. Therefore, in many countries, medical professional societies have proposed different access standards for the performance of laparoscopy and robotic surgery. These standards are based on the annual volume of specialized surgery in the hospital, the quantity and quality of surgeons' past open surgeries. Only the surgeons who meet the standard may perform laparoscopic or robotic surgery. A surgeon with experience in conventional surgery who learns to perform laparoscopic or robotic surgery will have a shorter "learning curve" and thus inflict less harm than those who directly go through training via laparoscopic or robotic surgery. Rigorous surgical training is the foundation of all modern technologies; there are no shortcuts.

Second, surgeons should select new surgeries or treatments for different diseases or distinct stages of the same disease. Selecting a suitable treatment is not only a technical issue but an ethical one. On the one hand, hospitals, industries, and insurance companies expect to develop and to promote high-tech surgeries. On the other hand, surgeons are always willing to try new techniques which challenge their limits. This continuous improvement of their techniques gives surgeons confidence and satisfaction after each success, which is also one of the main driving forces for the rapid development of laparoscopic and robotic surgery. Typically, surgeons choose the surgical methods that they are most familiar with, but once a surgeon comprehends more than three surgical techniques, 
one can choose the best treatment according to the nature of the lesion, the anatomical characteristics, and the needs of the patient. Nowadays, while ensuring the safety and the benefit of patients, clinical research in open, laparoscopic, and robotic surgery is mostly limited to the comparison of surgical safety and short-term efficacy after surgery. Little is known about the long-term outcome after surgery.

In 2018, doctors from MD Anderson Hospital published two studies on the long-term efficacy of minimally invasive surgery, such as robotic surgery, in uterine cancer in The New England Fournal of Medicine. One study was a phase III study that included 631 cases involving 33 medical centers around the world. However, it was suspended because it found that the 4.5 -year tumor recurrence rate of uterine cancer after minimally invasive surgery was significantly higher than that of traditional open surgery (2). Another study is a retrospective, epidemiological study of two major cancer databases, the National Cancer Database (NCDB) and the National Cancer Institute (NCI), in collaboration with Harvard University, Columbia University, and Northwestern University (3). The results showed that at 4 years after surgery, the risk of death was $9.1 \%$ for minimally invasive robotic radical hysterectomy and $5.3 \%$ for open radical resection. Meanwhile, the 4-year survival rate after minimally invasive radical resection dropped by $0.8 \%$ per year from 2006 to 2010 . It was concluded that minimally invasive surgery reduced the longterm survival of patients who underwent radical resection of early cervical cancer.

The results of these two studies changed the treatment plan and disease management of patients with early cervical cancer at MD Anderson Hospital. Although the negative results were influenced by multiple factors, it made us realize that laparoscopic technology with rapid recovery after surgery may benefit patients in the short term, but it is the long term that is more important. The long-term efficacy of laparoscopic surgery in other specialties is still being broadly evaluated. Therefore, before any conclusion is reached, surgeons should follow the guidelines with corresponding surgical indications.

Currently, in different countries, the ratio of laparoscopic or robotic surgery to traditional surgery varies between different medical centers. This variation is related to the economic development that affects equipment conditions and is related to the knowledge of medical decisionmakers based on the level of their surgical skills. In regular hospitals, taking hepatopancreatobiliary surgery as an example, only $20 \%$ of complex surgeries are performed by laparoscopic and robotic surgery, but the percentile might be $70 \%$ or even higher in some experienced specialty centers. This phenomenon may answer the core question of whether the patient or the surgeon actually needs new techniques in surgery. When surgeons are keen to learn new technologies, the "learning curve" is unavoidable, especially in complex hepatopancreatobiliary surgeries. For example, in the beginning, the average operation time of the laparoscopic Whipple procedure exceeds 10 hours. Many clinical retrospective studies have shown that the mortality rate of the laparoscopic Whipple procedure in hospitals with low surgery volume is significantly higher, which means that when surgeons are learning this new surgical technique, the price might be patients' lives.

In contrast, 30 years ago, at the beginning of laparoscopic cholecystectomy, the "learning curve" may have only increased accidental bile duct injuries rather than surgical mortality. Therefore, it is suggested that complicated laparoscopic and robotic surgery should be performed by experienced surgeons at specialized surgical training centers. These ideas are consistent with surgical development, and they can maximize the benefits for patients. Based on the current global imbalance in the development of surgical techniques, in developed countries and regions, the number of great medical centers specializing in laparoscopy and robotic surgery will rise. However, in developing countries, traditional surgery will still lead. Even with the rapid development of artificial intelligence, it can be predicted that this imbalance will continue for a long time. Therefore, improving traditional surgical techniques by integrating high tech is essential and might be the optimal solution.

Third, be rational with the development of high-tech surgery. Nowadays, a considerable amount of artificially intelligent high tech is evaluated in a "vacuum" state, so it might be difficult to replicate performances. When we see the success of AI in autonomous vehicles, we start to wonder if AI surgical robots will follow shortly, allowing surgeries to be performed autonomously. However, hightech devices are often built on standardized ideal conditions. For example, the premise of the autonomous vehicle system is that traffic rules are followed, and roads are in a standard setting. For AI surgical robots, a good full view and an organ with standardized anatomy are needed. The shortcomings of these recent technologies will limit their applications.

In February 2015, a patient in the UK died from the failure of robotic heart valve repair. At the later hearing, the surgeon admitted that "the application of this technology 
is a thing that any innovative surgeon would do." (4). However, the surgeon had performed the surgery too soon. Metaphorically, he had wanted to run before he could walk. Also, he should have informed the patient that the surgery was the first robotic surgery he had ever performed and that a traditional open surgery might be safer.

All of this serves as a warning to us for the application of novel technology. Accidents might happen beyond the first design. New techniques may bring unfamiliar problems that must be discovered and addressed during their applications. Therefore, there are new generations of equipment that make laparoscopy and surgical robots better. However, let us go back to the initial question: if the new and expensive equipment and techniques cannot improve the long-term efficacy compared with traditional surgery, are they still the techniques that we expect to learn and develop? The answer is simply: innovation is endless. Whether the public supports it or not, these technological innovations will continue to rise. What we need to do is to keep our minds clear and not being trapped by novel technology, so that we can continuously improve the long-term efficacy of surgical treatment while embracing new techniques.

\section{Acknowledgments}

Funding: None.

\section{Footnote}

Conflicts of Interest: The author has completed the ICMJE uniform disclosure form (available at https://hbsn. amegroups.com/article/view/10.21037/hbsn.2020.04.09/coif).

Cite this article as: Geng XP. How to balance the development of new surgical techniques and protection of patients' health interests. HepatoBiliary Surg Nutr 2020;9(3):333-335. doi: 10.21037/hbsn.2020.04.09
The author has no conflicts of interest to declare.

Ethical Statement: The author is accountable for all aspects of the work in ensuring that questions related to the accuracy or integrity of any part of the work are appropriately investigated and resolved.

Open Access Statement: This is an Open Access article distributed in accordance with the Creative Commons Attribution-NonCommercial-NoDerivs 4.0 International License (CC BY-NC-ND 4.0), which permits the noncommercial replication and distribution of the article with the strict proviso that no changes or edits are made and the original work is properly cited (including links to both the formal publication through the relevant DOI and the license). See: https://creativecommons.org/licenses/by-nc-nd/4.0/.

\section{References}

1. Altman LK. THE DOCTOR'S WORLD; When Patient's Life Is Price of Learning New Kind of Surgery. The New York Times 1992-06-23.

2. Melamed A, Margul DJ, Chen L, et al. Survival after Minimally Invasive Radical Hysterectomy for Early-Stage Cervical Cancer. N Engl J Med 2018;379:1905-14.

3. Ramirez PT, Frumovitz M, Pareja R, et al. Minimally Invasive versus Abdominal Radical Hysterectomy for Cervical Cancer. N Engl J Med 2018;379:1895-904.

4. Droese R. UK's first robot-assisted heart valve surgery results in patient's death. The New York Times, 1992-0623. RT news, 2018 Dec. 